\section{On Dynamic Geometry Software in the Regular Classroom}

\author{
Thomas Gawlick, Bielefeld
}

\begin{abstract}
The availability of Dynamic Geometry Software (DGS) in the regular mathematics classroom has the potential of enriching and furthering the learning process, but also includes the danger of pitfalls due to peculiarities of Dynamic Geometry as well as side effects of the interactivity. In view of the forthcoming large scale introduction of DGS, it seems desirable to estimate its net outcome in terms of the influence on the learners' average achievement. Before elaborating on the shortand medium-term influence of DGS and differential effects of gender and achievement level, we position our approach in the research field by giving a synopsis of previous investigations on the impact of DGS in the classroom.
\end{abstract}

Kurzreferat: Die Verfügbarkeit von DGS im regulären Mathematikunterricht birgt einerseits das Potential zur Förderung und Bereicherung des Lernprozesses, andererseits aber auch Fallstricke in Gestalt von Eigenheiten der Dynamischen Geometrie und Nebenwirkungen der Interaktivität. Angesichts der bevorstehenden flächendeckenden Einführung von DGS erscheint daher Aufschluss darüber wünschenswert, was sie „unterm Strich“, also bzgl. des mittleren Lernerfolgs bewirkt. Bevor wir dies näher hinsichtlich kurz- und mittelfristiger Effekte beleuchten und nach Geschlecht und Leistungsstand differenzieren, verorten wir das gewählte Design im Forschungsumfeld durch einen systematischen Abriss früherer Zugänge zur Untersuchung des DGS-Einflusses im Unterricht.

ZDM-Classifikation: C30, C70, G10, N80, U70

\section{Introduction}

13 years after the introduction of "Cabri géomètre", Dynamic Geometry Software (DGS) is about to make its way into the mathematics classroom. Its various capabilities are widely believed to support learners in gaining insights and developing heuristics. But qualitative analyses of learning processes with DGS clearly show the double-edged character of computer application: on the one hand a mathematically extremely attractive extension of scope due to its visual and heuristic potential - on the other hand the danger that learning "DGS geometry" means creating new subjective domains of experiences and that interactivity entails "degoaling".

Therefore it seems appropriate to investigate the impact of DGS on students' achievements, conceptions and attitudes in the regular mathematics classroom. In this paper, we present the results of our recent study concerning DGS' differential effects on students" achievement.

\section{Previous research}

The development of empirical research on (DGS) in the classroom may be divided into three stages:

1. the development and testing of appropriate teaching units,

2. enquiries concerning their local / short-range effects,

3. consideration of their more global /medium- or even long-range effects.

Of course these stages are not to be seen as separate as the acts of a play but rather as a perhaps necessarily repetitive cycle of innovation - though one can easily recognise an upward shift in the focus of activities in the whole field as well as in subsequent publications of individuals or research groups.

In the first stage, researchers were mainly concerned with the exploration of the various capabilities of DGS and their integration into the teaching process. Consequently, pioneers' papers like Laborde (1992) or Laborde \& Sträßer (1990) mainly focused on the really promising potentials of the new technology rather than on the impact caused by it in the classroom, though they already took the influence of the software on the solution of tasks into account. The apparent focus on features of the software certainly also stems from the fact that the introduction of computer technology in mathematics education was closely linked to the "microworld approach" to learning: "The essential idea is that through play within a microworld, children come to understand its features - features which have been 'planted' according to a priori learning objectives. ... Thus, microworlds can be viewed as computational environments which 'embody' mathematical ideas." (Hoyles et al. 1991) This especially holds for DGS, as Laborde \& Laborde (1995) point out: "Cabri-Géomètre provides a 'real' model of the theoretical field of Euclidean geometry in which it is possible to handle in a physical sense the theoretical objects which appear as diagrams on the screen." By dragging their constructions, students learn to distinguish "diagrams drawn in an empirical way from diagrams resulting from the use of geometric primitives." (ibid.) Thus, the concept of "messing up" in combination with the concept of direct manipulation were seen as apt to let students experience the meaning of the geometrical concept of constructions. This should entail "that much if not all of the knowledge schools presently try to teach with such pain and expense and such limited success will be learned, as the child learns to talk, painlessly, successfully and without organised instruction." (Papert 1980)

However, in a critical review of the role attributed to DGS in the literature, Hölzl (2001) concludes that though a lot of examples are given how DGS can support the heuristic phase of problem solving, a closer look leads one to question whether the software is really used in a methodical and activating way of knowledge acquisition. Rather, it seems that DGS is used in a solely verifying manner: i.e. the learners need only vary more or less prefabricated geometric configurations to confirm empirically more or less explicitly stated conjectures. (In fact, verifying the intersection properties of triangle transversals or the properties of the Euler line are often mentioned as examples - even recently in the computer 
math column of a renowned research journal.)

In the same vein, a second stage of development of research on DGS in the classroom revealed a somehow diverse image by a closer look on what really took place in the students' interaction with the computer: On the one hand, in their studies on the mediating function of several computer-based learning environments Noss \& Hoyles (1996) frequently observed that learners recognised mathematical structures and relations in the patterns of a specially designed microworld. But on the other hand, there was increasing evidence that the knowledge constructed thereof did not fit neatly into the mathematical structures aimed at but was rather closely linked to the specific environment in which it was developed: The qualitative empirical studies by Hölzl were path-breaking: Hölzl $(1994,1996)$ investigated the interaction of students with DGS. By interpreting transcripts of videotaped problem-solving processes of student pairs in a required elective course, he aimed at empirically relating didactical analyses of the content as presented via DGS with the actual course of students' problem-solving processes. He shows that the interactive possibilities of the medium motivate the students to develop individual interests and stamina. The software causes, however, subtle interference between the intended understanding of geometry and the understanding the students acquire. This gave rise to the suspicion that the use of DGS leads to the genesis of new subjective domains of experience in the realm of geometry instead of an elaboration and reinforcement of the ones acquired in the classical paper and pencil environment (Graumann et al. 1996) ${ }^{1}$. Furthermore, Hölzl observed the following problematical features of students' interaction with DGS:

* Avoidance of mathematical analysis: the students are oriented towards the practical implementation of a solution and not towards its theoretical connotations and implications.

* Circumvention of the application of tools and preference of simpler concepts.

* Non-reflected utilization of tools.

* Deviation from the given objectives.

Due to the chosen design it remains open, however, to what extent these aspects are really caused by using DGS or rather by the teaching unit, the teacher himself or other influences. But other evidence supports that DGS does change the working style of students: For instance, Healy $\&$ Hoyles (2001) report a teaching experiment that lead one successful pair of students to 'web' their solution process using the DGS tools and to take even the step from argumentation to deduction - whilst in the work of ten less successful students the use of DGS tools did not fit with the students' strategies, even if they were more promising mathematically. A closer look at external factors influencing the outcome of DGS based learning processes is thus in order.

Therefore, in a third stage of research, the perspective had again to be broadened. Noss \& Hoyles (1996) describe, how, in the transformation of innovation "from microworlds to schoolworlds", the original momentum of

\footnotetext{
${ }^{1}$ It was also argued that the geometry embodied by DGS was
} not necessarily the traditional Euclidean one. innovation somewhat fades away during a process of iterated mediation of content under external constraints: the teachers' aims and beliefs as well as the necessity of negotiations for hardware availability and guaranteeing compatibility with traditionally taught courses. One particular problem on the side of teacher is the fear to lose control over what the students do. This leads to a rejection or circumvention of the openness of the original microworld approach.

Hölzl (1999) reports on the long-term application of DGS in a classroom where DGS was an integral part of the learning environment and states the following results:

* DGS possesses a considerable heuristic potential in the connection of transformation geometry.

* The application of DGS should only be realised after thorough consideration: "Dynamics per se is not a didactical advantage."

* The intensive application will be most favourable where an objective instrumental requirement meets advanced mathematical teaching experience.

* The drag mode can be used

$$
\text { as a graphic tool, }
$$

as test mode,

as a search mode.

* The use of drag mode as a test mode remained shaky even after two years.

Hölzl's distinction between different ways of using the drag mode shows up in Arzarello et al. (1998) in refined form: in the "search mode" they distinguish "wandering dragging" (without definite aim) from "lieu muet dragging" (following an invisible locus where a property relevant to the particular problem is fulfilled). But obviously it is a challenge for the teacher to provide suitable material and feedback for the students to help them in unfolding their usage of the drag mode gradually - according to Hölzl (2001), finding ways to accomplish this is a "significant but difficult research task".

Having observed the development of teaching sequences over a period of three years, Laborde (2001) identifies subsequent steps in the integration of DGS into secondary mathematics teaching. The software gradually changed its role from a "visual amplifier" or provider of data to an essential constituent of the meaning of tasks. In this last and essential stage technology also begins to affect the meaning of the taught concepts. This requires a change of perspective on geometrical objects that is not easily obtainable. According to Laborde, this accounts for one part of the observed difficulty of integrating technology in the classroom. A second part arises from the necessity to integrate DGS in such a way that the students do profit from it in terms of the official curriculum. But obviously its content is not necessarily well-suited to interrelate with the new conceptual aspects introduced by virtue of technology's benefits. In the teaching processes described by Laborde DGS finally became a reference for official knowledge. Whether this can be accomplished seems to be the criterion for a successful integration of technology in the sense of Laborde: The necessary reinforcement of the links between mathematics and technology was considered as "final proposal for the definition of integration: technology gives a meaning to mathematics and 
mathematics justifies the use of technology." (loc. cit., p. 316)

Laborde observed that in order to achieve this outcome, one has to deal with some problematic beliefs of the teachers concerning the way students learn mathematics and how it has to be presented to them:

Dichotomy between conjecture and explanation: DGS is only used in the empirical confirmation of conjectures but not in the process of proving them,

Repetition of tasks: the same tasks were presented in both environments though on paper they may be less demanding because of the possibility to solve them by perceptive strategies instead of constructions. This fits into the results of an enquiry about the assumptions of undergraduate students on the use of technology. Povey \& Ransom (2000) summarise their attitude as "doing maths by hand indicates that one understands it". This seems to be connected to the fact that paper and pencil is often understood as "not a context" so that its use instead of technology substantiates "real understanding" in the sense of a successful decontextualization of the acquired knowledge. In contrast to this, Noss \& Hoyles (1996) introduce a concept of situated abstractions: "In computational environments, there can be an explicit appreciation of the form of generalised relations within them ... while the functionality and semantics of these invariants - their meanings - is preserved and extended by the learners" (p.125). According to Laborde (2001), the linkage of understanding with paper and pencil achievement may also be explained by the institutional constraint that examinations are solely given in a paperand-pencil environment.

Complexification of tasks: In the beginning of technology's integration, the rich feedback of DGS easily leads teachers to propose DGS tasks of greater complexity than on paper. They presuppose that using DGS solely facilitates the solution process - but the use of technology itself as well as students' specific reactions on it recurrently cause unforeseen difficulties. Related to this, it seems plausible that teaching innovation invariably entails time inflation (the factor 4 is reported by Laborde as well as by Schneider 1999).

Laborde also reports the steps taken by the observed teachers in order to fully incorporate technology into the mathematics classroom:

Some homework and a part of the usual assessment were based on DGS: Using DGS for homework was a means to give students the possibility to develop their own "schemes of instrumentation" of the tool at their own pace; using DGS for assessment served to give legitimacy to the instrumented solutions,

Mathematical contents were institutionalised in relation to technology: this means that DGS was allowed as a reference context for some of the properties, theorems or definitions that form the corpus of the official knowledge (formulated by the teacher, but supposed to be shared by or at least understandable for all students of the country), making it possible for the students to exploit the full power of DGS as a tool for the mediation of theoretical constructs without changing the reference context.

However it should be stressed that these steps emerged only after one or two years from a somehow evolutionary process on the side of the teachers (and probably also of their classes). It seems probable that one cannot just start with this stage of integration but rather has to develop it gradually.

\section{Aims and layout of the present study}

At the time being, DGS is set to be included in the curriculum and the school authorities have teachers being trained on it. Consequently, it seems adequate to examine in regular classrooms the impact of DGS on the learners' results. Of course, this impact can only be judged in comparison to the same teaching in the paper-and-pencil environment. From media research it is known that the choice of medium is far less important than the nature of the treatment (see Salomon 1978 and Clark 1983). Consequently, we decided to have the same teachers giving the same lessons in both environments. Using the same tasks was also in accordance with Laborde's above mentioned observation that teachers continue to repeat DGS tasks on paper. It is to be expected that the change of medium will not immediately entail a change of tasks (though this may prove to be more appropriate in the long run), due to curricular reasons as well as to accomplish a gradual change in teaching.

With the background of research presented above, we were in the position to investigate how the step from experimental to regular DGS use will probably take place in the classroom. The following factors had to be taken into account:

1. Selection and processing of appropriate material.

2. Training of teachers on how to make use of it.

3. Alignment of the developed teaching sequences with regular teaching conditions.

1. As observed by Laborde (2001), integration of technology will go along with a shift of tasks. But other than Laborde, we could not accompany teachers' familiarisation with DGS over a longer period. At present this corresponds to the situation at schools (at least in Lower Saxony), where teachers have to cope with the integration of DGS into their teaching without much previous preparation. In such a situation it is a common practice to draw on experts' material. We chose to accomplish this by examining the use of interactive electronic working sheets. Three reasons account for this decision:

Didactical advantages: The concept of Elschenbroich \& Seebach (2000) can be summarised as "From programming new figures to exploring given ones!" and it can draw on several years of developmental and evaluative work of its proponents. It seems appropriate to overcome some of the difficulties observed when starting with a blank screen. More specifically, not much time is necessary to train students on DGS, since they need not construct - it suffices to set points, connect them by straight lines, drag marked points and draw loci. This should help to prevent students from "degoaling" as well as from creating new subjective domains of experience. And last not least: the used material allows for an experimental rather than a solely verifying use of DGS.

Ecological validity: In comparison to material 
presented at teacher-training workshops, these worksheets fit neatly into the standard curriculum and are developed to a "ready to use" stage even for novices. So we expect that many teachers who are novice to DGS or get only some training by a local "multiplicator" will resort to such worksheets.

Methodological advantages: In a comparative study, one has to equalise the training material of the students. Due to the chosen design, one can control that the electronic worksheets resemble their paper versions as close as possible.

2. For the 12 lessons to be given, teacher students replaced the usual teachers of the experimental classes. Together with them, we developed a series of lessons and had the teacher students in advance been trained intensively on our courseware. Additionally, the material was connected to the textbooks in use.

3. Of course we had to observe the time constraints of the regular curriculum as well as the availability of the schools' computer laboratories. This limited the DGS quota to max. $30 \%$. In addition to that, we had to refrain from electronic homework or assignments. These restrictions will probably also hold in other schools - at least for the next years.

Finally we had to decide upon our method of evaluation. To avoid the known critique of qualitative studies on individuals or single classes as not being representative we chose a broader basis of nine classes. As a consequence of this, we were mainly confined to quantitative methods, though we audiographed and analysed several key lessons. According to a detailed meta-analysis, Ruthven (1997) stated that only a small number of studies regarding computer algebra had an acceptable design (i.e. experimental and control groups, pre- and post-tests), which we then incorporated as follows:

\section{Method}

Procedure The study was performed in nine classes (grade 7; number of students $\mathrm{N}=214$ ) of three senior high schools. At each school, three classes were assigned to the $\mathbf{C}$ (omputer), $\mathbf{P}$ (aper) and $\mathbf{V}$ (control) group respectively yielding the following hierarchical design:

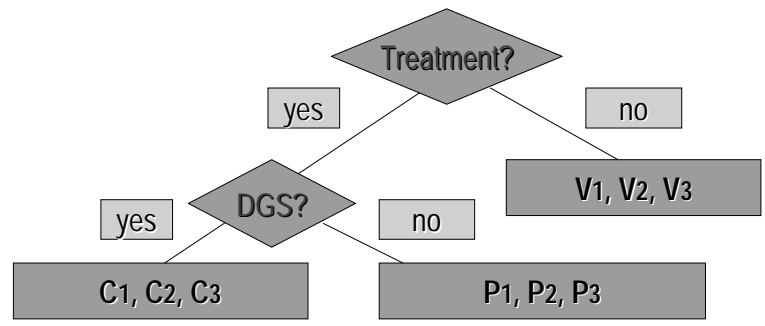

Pre- and post-tests were performed in all classes. The pretest surveyed the prior geometric knowledge of the students as well as their attainment levels. The three tasks were: equal distribution of a pizza slice, completing the half of a butterfly, reconstruct the marks for the kick-off and the goals on a football field.

Treatment The treatment covered perpendicular and angular bisector, the circumcenter and the circumcircle, the incentre and the incircle of triangles. In $\mathrm{C}$, we used interactive electronic work sheets (cf. Elschenbroich 2001) that focus on investigating given figures rather than constructing new ones. As an extra dimension, we embedded geometry tasks into problem contexts: for instance the equal distribution of soil was posed as a problem (compare fig.1) to introduce a small modelling process ending up with the necessity to find a construction for bisecting angles - thus demonstrating the usability of mathematical tools in "real life".

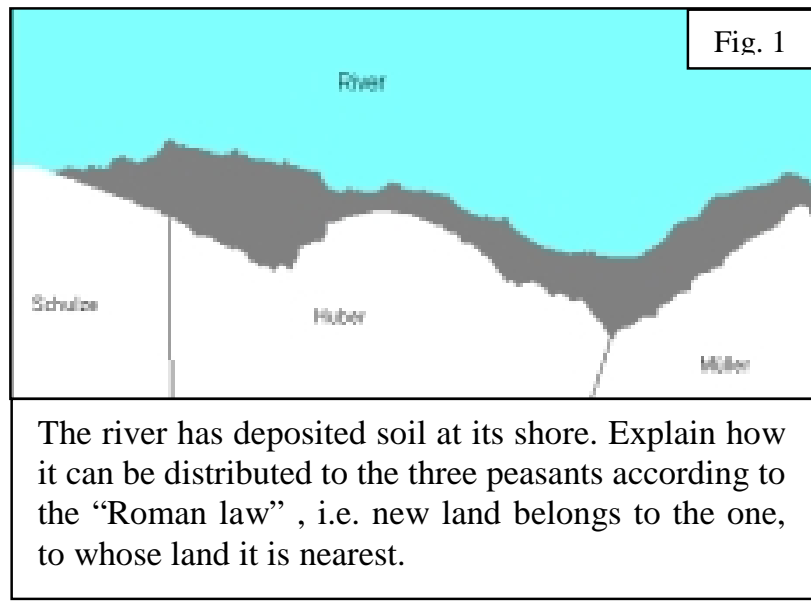

The treatment was implemented in the experimental groups using the same work sheets in the respective learning environment (i.e. paper and pencil in $\mathrm{P}$ resp. DGS in $\mathrm{C}$ ). At every school, the $\mathrm{C}$ and $\mathrm{P}$ classes were taught by the same teacher student. Ordinary geometry lessons took place in the $\mathrm{V}$ classes.

Post-test After the treatment, a post-test was performed in the form of a written examination lasting 45 minutes. Topics were partly reproductive (explaining the construction of perpendicular bisectors and the distribution of soil), partly reorganizative: we repeated the football field task (to have a parallel item and to see whether strategies were changed) and included one task (see fig. 6) to especially account for a possible influence of dragging: will students be more able to imagine the movement of the circumcentre if they have already seen intersection points moving?

Follow-up After six months, the post-test was repeated in all classes. As an extra task, a variant of the circumcentre task was included to see whether students were able to transfer their knowledge to other applications after some time.

Measurement The learners' achievement was measured by the pre-post-difference, which was checked against mean post-test achievement adjusted by pre-test. The tests can be divided into procedural and explanatory parts, so the pre-post-difference $\mathrm{D}$ splits into a procedural component $\mathrm{D}_{\mathrm{p}}$ and an explanatory component $\mathrm{D}_{\mathrm{e}}$ :

$\mathrm{D}=\mathrm{D}_{\mathrm{p}}+\mathrm{D}_{\mathrm{e}}$.

Hypotheses

1) The problem-based approach significantly influences the achievement.

2) The use of DGS does not significantly influence the achievement.

Nevertheless we expected that DGS would influence the chosen strategies! 


\section{Results of the post-test}

The box plot of the pre-post-difference $\mathrm{D}$ and its procedural component $\mathrm{D}_{\mathrm{p}}$ in fig. 2 reveals:

* Overall outcomes were about equal in $\mathrm{P}$ and $\mathrm{C}$ and considerably higher than in $\mathrm{V}$, with smaller variance in $\mathrm{P}$.

* In the procedural parts, students in $\mathrm{P}$ did somewhat better than in $\mathrm{C}$ and $\mathrm{V}$, with considerably greater variance in $\mathrm{V}$.

* All in all, C lay due to the lower second quartile a little behind $\mathrm{P}$ (regarding both measures).

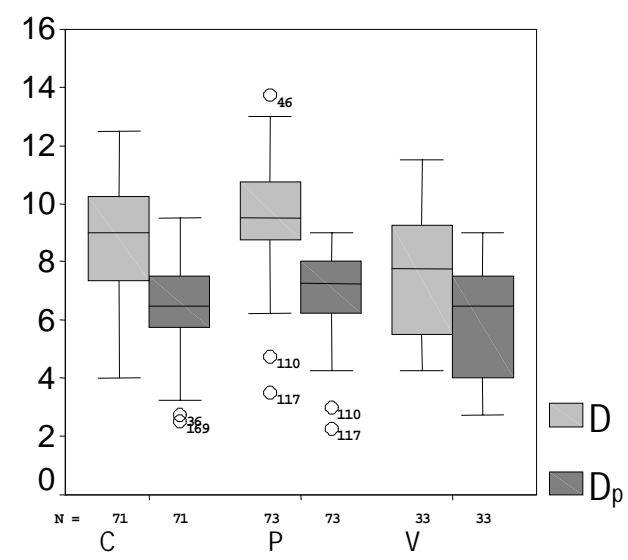

Fig. 2

Inference statistics yields somewhat heterogeneous results: In the whole population we find for $\mathrm{D}$ that $\mu(\mathrm{P})$ $\gg \mu(\mathrm{C}) \approx \mu(\mathrm{V}), \mu(\mathrm{P} \cup \mathrm{C})>\mu(\mathrm{V})$, so 1$)$ can be confirmed, 2) has to be rejected - but this result is slightly obscured by the fact that the means of $\mathrm{P}$ and $\mathrm{C}$ differ only by .75. This difference is significant $(\alpha=.05)$, but is less than the insignificant difference 1.00 of $\mathrm{C}$ and V. So one might suspect a statistical artefact due to the diverging group sizes (the drop-out rate was -not uncommonly- significantly higher in V).

An explorative data analysis is thus in order. For practical purposes, we restricted ourselves to factors that are easily accessible to teachers: Besides gender, we distinguished higher and lower achievers by splitting at the median (this procedure has also statistical advantages). Here, we have to restrict ourselves to presenting some tendencies in the data (fig. 3-5; for more details see Gawlick 2001a):

* Except for girls at public schools, in C classes higher achievers seem to profit more than lower achievers, vice versa in $\mathrm{P}$,
* While in public schools $\mathrm{P}$ and $\mathrm{C}$ scores are about equal, for girls in the private schools $\mathrm{P}$ scores were (significantly) higher than $\mathrm{C}$ scores,

* For girls at public schools, C appeared to be slightly more effective than $\mathrm{P}$, irrespective of their achievement level.

1. This is a campground from above. The friends Anton, Bert and Charley want to have breakfast together. Can you place a table on the campground in equal distance to their tents?

2. What happens if Charley moves his tent along the sea to find the best fish ground? Why?

3 . If he chooses a certain place for the tent, a problem will occur to the three friends. Can you figure it out?

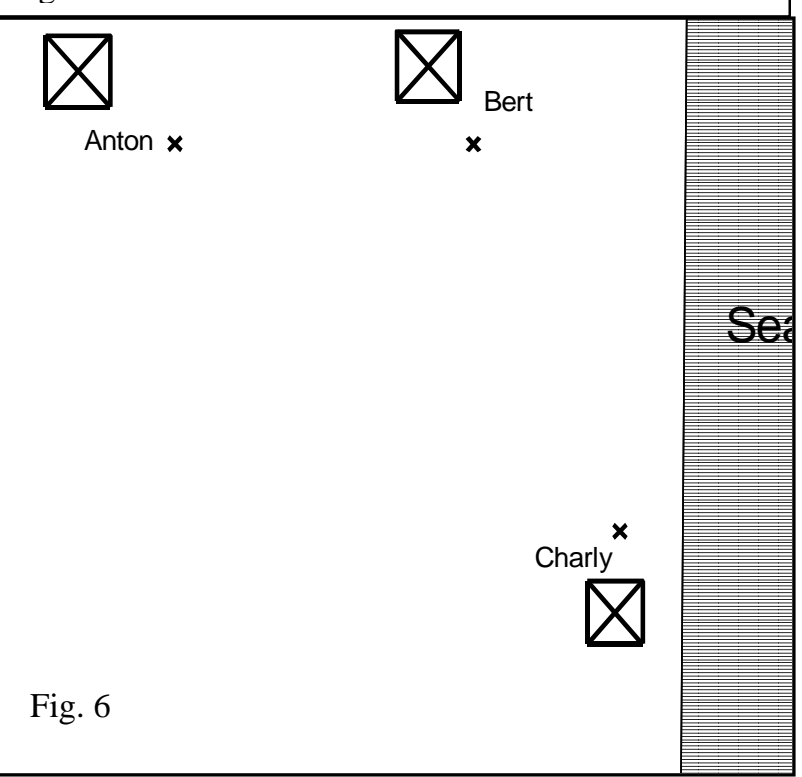

Also, differences in achievements and strategies between $\mathrm{P}$ and $\mathrm{C}$ occurred with "dynamic" problems (but surprisingly also with some "static" ones like the construction of perpendicular bisectors). The post-test contained one task to account especially for the dynamics (see fig. 6).

Part 1 involves the utilization of the circumcentre in a problem context. To see whether DGS was helpful in developing dynamic mental images, students were asked question 2. This offered an opportunity to check whether students realized the limitations of such a mathematical modelisation in question 3. All in all, the "dynamic task"

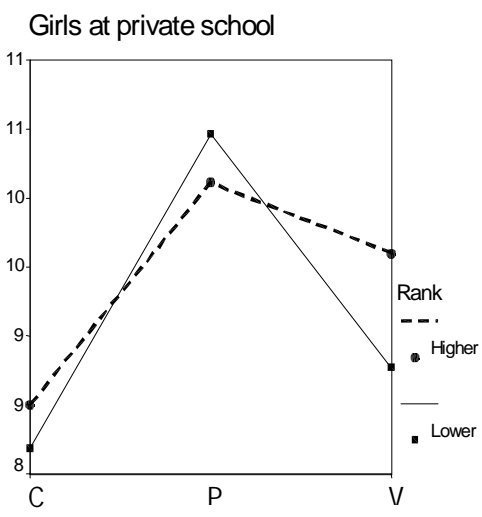

Fig. 3

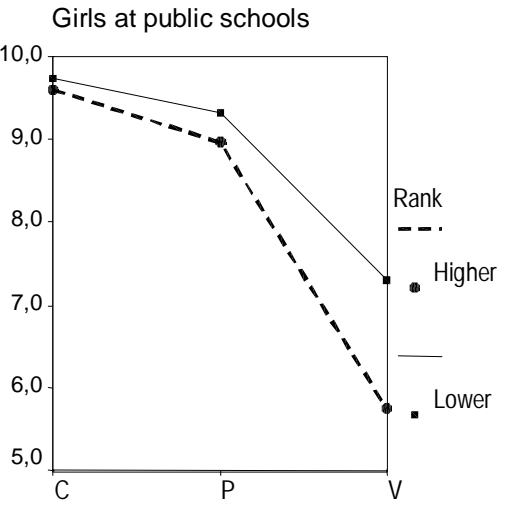

Fig. 4
Boys at public schools

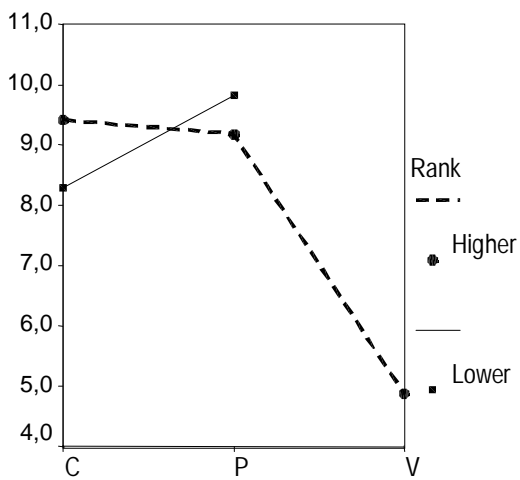

Fig. 5 
2 is certainly apt to show differences in outcome if DGS is really more than "just another medium". Again, the results are rather ambiguous:

* The total score for the "dynamic task" does not show any significant differences.

* If one considers only the students' hypothesis about the movement of the table, $\mathrm{C}$ is clearly ahead of $\mathrm{P}$,

$*$ and vice versa if one considers their explanations why this will happen.

These adverse effects nearly cancel out each other. A possible interpretation would be: DGS is helpful to generate hypotheses, but not to find arguments supporting them. This would fit neatly to the ,degoaling“-effects observed by Hölzl (1996) and the findings of Lewalter (1997) concerning learners' superficial understanding of animated illustrations.

\section{Results of the follow-up}

In what follows, we compare the differences between preand post-test (PP) to the differences of pre-test and follow-up (PF). Because the follow-up contained an extra task, the latter ones are expected to be higher.

\begin{tabular}{|c|c|c|c|c|c|}
\hline Difference & Group & $\mathbb{1}$ & & Mean & $\begin{array}{l}\text { Standard } \\
\text { deviation }\end{array}$ \\
\hline \multirow[t]{2}{*}{$\mathrm{PP}$} & & 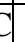 & 62 & 8,9435 & 1,9425 \\
\hline & & P & 62 & 9,7339 & 1,7683 \\
\hline \multirow[t]{2}{*}{$\mathrm{PF}$} & & $\overline{7}$ & 62 & 12,6774 & 5,1091 \\
\hline & & P & 60 & 12,5250 & 5,0956 \\
\hline
\end{tabular}

This statistics only considers students that took part in all three examinations $(\mathrm{N}=124)$. Results are as follows:

* In the follow-up the $\mathrm{C}$ groups now score slightly higher than $\mathrm{P}$.

* The difference between $\mathrm{P}$ and $\mathrm{C}$ groups diminished in the follow-up.

We use explorative data analysis to find possible explanations. We compare again higher achievers (ranking 1) to lower achievers (ranking 2) and boys (population 1) to girls at public schools (population 2) and girls at the private school (population 3), so in all we distinguish six subgroups. Fig. 7 shows how the percentage of correct solutions varies with time in these subgroups (note that due to experimental mortality in the control group there were no higher achieving boys that took part in the follow-up):

* In the groups of coeducated boys and separately educated girls higher achievers remember more in $\mathrm{P}$ classes than in $\mathrm{C}$ classes.

* In contrast to this, higher achieving coeducated girls profit more in $\mathrm{C}$ classes than in $\mathrm{P}$ classes,

* Amongst the lower achievers, the differences are less remarkable and tend to fade out with time.

\section{Discussion}

It seems quite plausible that DGS lay behind in the shortterm comparison:

* In C classes, some time had to be spent for computer handling.

* Especially for lower achievers, even the mere dynamic presentation of the usual geometric figures may have been an extra learning content

* Lower achieving boys were observed to misuse DGS as a play-thing.

For the medium-range advantage of DGS may account that

* to assimilate DGS experiences and reproduce them with paper and pencil requires a mental reorganization that may have furthered the remembrance especially of higher achievers,

* higher achieving girls were observed to profit extraordinarily from the change between experimental group work (where girls worked always together with girls only) and class discussion.

Given that, the results concerning the private school do surprise a little bit, but may partly be explained by the
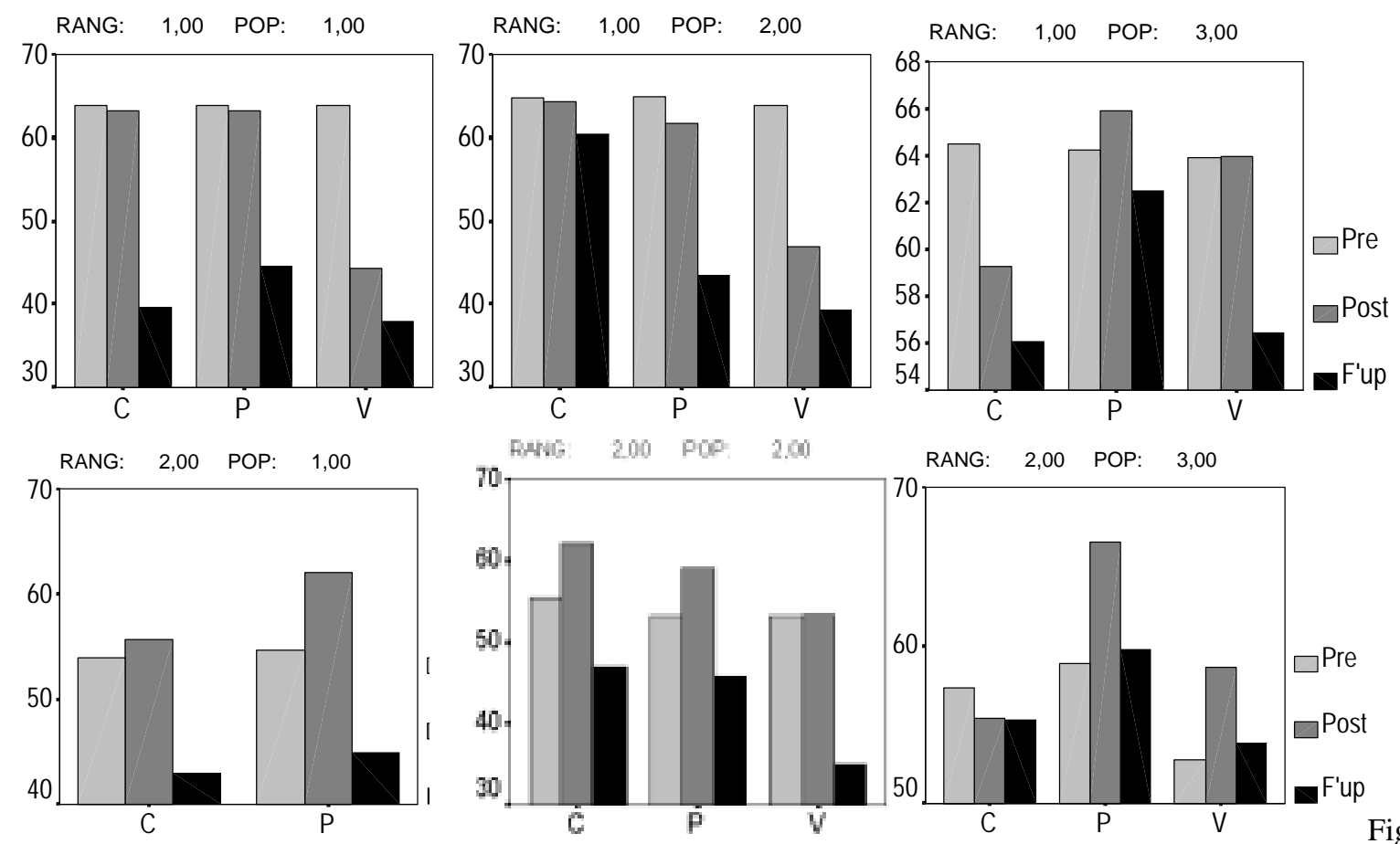

Fig. 7 
fact that in this environment homogenous group work could not make such a great difference because there were no boys. On the other hand, the deviation of the private $\mathrm{P}$ group should also not be overestimated: this group was a rather extraordinary class (including the two best students of the whole sample) that seemed to develop even better during the treatment. We concentrate thus on explaining the results at public schools.

Our main point is: The medium-term advantage of the $C$ groups seemingly originates from the better performance of higher achieving girls in these groups.

This conclusion is supported by the following facts:

1. Statistically, the difference between $\mathrm{P}$ and $\mathrm{C}$ is significant only for co-educated girls $(\mathrm{t}=2,077$, $\mathrm{df}=48,775, \mathrm{p}=, 043)$, but not for boys $(\mathrm{t}=-, 333, \mathrm{df}=24,167$, $\mathrm{p}=, 742$ ).

2. If one additionally distinguishes higher and lower achievers, the only significant difference occurs for higher achieving co-educated girls $(t=2,688, d f=16$, $\mathrm{p}=, 016)$.

3. Comparing qualitative classroom studies, successful learning processes documented in the literature (e.g. Hölzl 2001) tend to occur among girls, whereas "degoaling" was reported from boys (Hölzl 1994).

For more details regarding results (and the implementation) of our study we have to refer to Gawlick (2002). However, one episode from our classroom observation should not be left unmentioned: One reason for restricting the scope of our treatment to standard material and for using prefabricated electronic worksheets was to avoid any possible confusion by deviations of Dynamic Geometry from the usual Euclidean one. To our surprise, such a "dynamic aberration" nevertheless occurred: In one of our schools we used the DGS "Cinderella" which was designed as "continuous" in order to avoid the disturbance of students by jumping points while dragging. This produced the following phenomenon: In fig. 8a, by dragging the vertices of the triangle students shall discover that its angular bisectors meet inevitably in one point I. However, it may be considered harmful (especially with regard to the interpretation of I as in-centre), that dragging vertex B through A lets "Cinderella" sometimes move I out of the triangle, as in fig. 8b. Of course this behaviour of "Cinderella" is apt to put the teacher into real difficulties (in the observed situation, our teacher student "solved" them by letting the students reload the worksheet).

However, it can not be easily dismissed as just faulty quite to the contrary: a mathematical analysis reveals that it is a mathematically necessary consequence of "Cinderella"'s continuity!

This fact strongly underlines the necessity for a background theory of dynamic geometry. For more

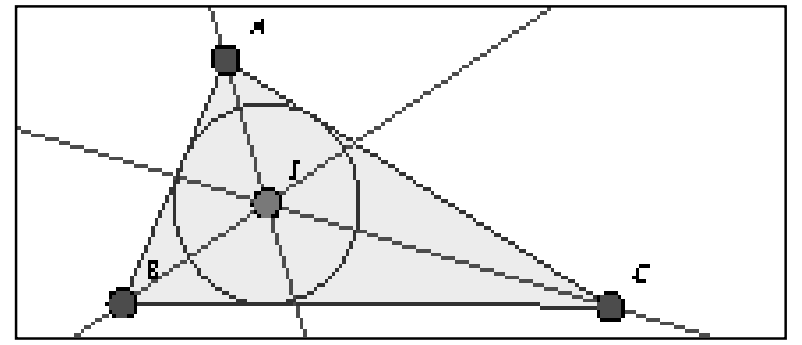

Fig. 8a details on this subject we have to refer the reader to Gawlick (2001, 2001b).

\section{Concluding remarks}

Our results show that one should not expect too much just from dynamizing the current curriculum. Probably this has to be the first step to integrate DGS into the classroom, but it should definitely not be the last one! We have seen that even if one opens the standard examples for exploration in a carefully designed course, the benefits of dynamics can easily be outweighed by the extra costs of DGS. So we strongly confirm that "dynamics is not a didactical advantage per se" (Hölzl 1999). The use of DGS should be preceded by thorough consideration and should certainly go beyond a mere change of media. For best results, one should consider a "dynamic restructuring" of the curriculum and admit DGS as a tool upon which the "official knowledge" can be based. It is necessary to change the educational environment accordingly, i.e. teachers must be put into a position to develop new teaching sequences, and schools must have the equipment to make DGS home work and assessment possible. As for the benefits of this process, our results indicate interesting perspectives on the differentiated furtherance of students in DGS based group work.

\section{References}

Balacheff, N. (1993): Artificial Intelligence and Real Teaching. In: Keitel, C.; Ruthven, K. (eds): Learning from Computers: Mathematics Education and Technology. Berlin: Springer, 131-158.

Clark, R. E. (1983): Reconsidering research on learning from media. Rev. Educ. Res. 53, 445-459.

Elschenbroich, H. J.\& Seebach, G. (1999): Dynamisch Geometrie entdecken. Elektronische Arbeitsblätter mit Euklid. Köln: Stam.

Elschenbroich, H.-J. (2001): Electronic Interactive Worksheets: A visual and dynamic way to learn geometry. Plenary talk, Cabri World 2001, Montreal. http://www.eiwos.com/indexelsch.htm

Gawlick, Th. (2001): Zur mathematischen Modellierung des Dynamischen Zeichenblatts. In: H.-J. Elschenbroich, Th. Gawlick, H.-W. Henn (eds.): Zeichnung - Figur - Zugfigur. Hildesheim: Franzbecker 2001.

Gawlick, Th. (2001a): Zum Erwerb geometrischer Grundbegriffe mit bzw. ohne DGS im regulären Mathematikunterricht. In: W. Herget, H.-G. Weigand, T. Weth (eds.): Lernen im Mathematikunterricht mit Neuen Medien. Hildesheim: Franzbecker 2001

Gawlick, Th. (2001b): Dynamic Notions for Dynamic Geometry. Proceedings of ICTMT5, Klagenfurt (in press).

Gawlick, Th. (2002): Zum Einfluss des Einsatzes von Dynamischer Geometrie-Software in anwendungsorientiertem Geometrieunterricht. In: Vechtaer hochschuldidaktische Forschungen und Berichte, Heft 6 (in press).

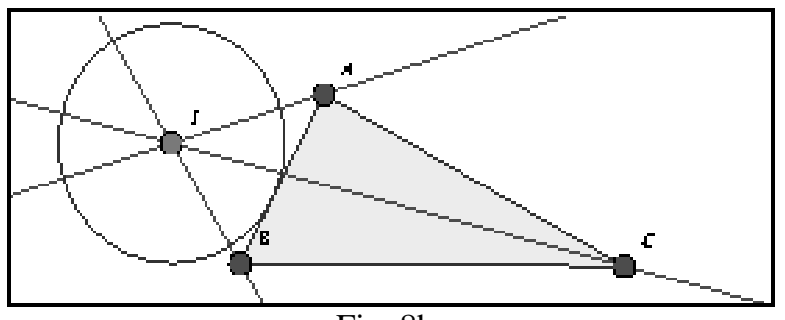

Fig. 8b 
Graumann, G. et al. (1996): Tendenzen der Geometriedidaktik der letzten 20 Jahre. JMD 17(3-4), S. 163-237.

Healy, L.; Hölzl, R.; Hoyles, C.; Noss, R. (1994): Messing up. Micromath 10(1), 14-16.

Healy, L.; Hoyles, C.; (2001): Software Tools for Geometric Problem Solving: Potentials and Pitfalls. Int. J. Computers for Math. Learn., vol. 6(3), 235-256.

Hölzl, R. (1994): Im Zugmodus der Cabri-Geometrie. Weinheim: Dt. Studien-Verlag.

Hölzl, R. (1996): How does 'dragging' affect the learning of geometry? Int. J. Computers for Math. Learn., vol. 1(2), 169187.

Hölzl, R. (1999): Qualitative Unterrichtsstudien zur Verwendung dynamischer Geometrie-Software. Augsburg: Wissner.

Hölzl, R. (2001): Using DGS to add Contrast to Geometric Situations - A Case Study. Int. J. Computers for Math. Learn., vol. 6(1), 63-86.

Hoyles, C.; Sutherland, R. (1989): Logo Mathematics in the Classroom. London: Routledge.

Hoyles, C.; Noss, R.; Sutherland, R. (1991): Final report on the Microworlds Project 1986-1989. London: University of London, Institute of Education.

Hoyles, C. (1993): Microworlds/schoolworlds: the transformation of an innovation. In: Keitel, C.; Ruthven, K. (Hg.): Learning from computers: Mathematics education and technology. Berlin: Springer, 1-17.

Laborde, C.(1992): Solving Problems in Computer Based Geometry Environments: The Influence of the Features of the Software. ZDM 24(4), 128-135.

Laborde, C.(2001): Integration of Technology in the Design of Geometry tasks with Cabri-Geometry. Int. J. Computers for Math. Learn., vol. 6(3), 283-317.

Laborde, C.; Laborde, J.-M. (1995): What about a Learning Environment where Euclidean Concepts are manipulated with a Mouse? In: A. diSessa et al. (eds.) : Computers for Exploratory Learning. Berlin: Springer.

Laborde, J.-M.; Sträßer, R.(1990): Cabri-géomètre: A Microworld of Geometry for Guided Discovery Learning. ZDM 22(5), 171-177.

Lewalter, D. (1997): Kognitive Informationsverarbeitung beim Lernen mit computerpräsentierten statischen und dynamischen Illustrationen. Unterrichtswissenschaft 25(3), 207-222.

Noss, R.; Hoyles, C. (1996): Windows on Mathematical Meanings. Dordrecht: Kluwer.

Papert, S. (1980): Mindstorms. Children, Computers and Basic Ideas. New York: Basic Books.

Povey, H.; Ransom, M. (2000): Some Undergraduate Students' Perceptionsof using Technology for Mathematics: Tales of Resistance. Int. J. Computers for Math. Learn., vol. 5 (1), 47 63.

Ruthven, K. (1997): Computer algebra systems (CAS) in advanced-level mathematics. University of Cambridge.

Salomon, G. (1978): On the future of media research. Educ. Comm. \& Techn., 26, 37-46.

Schneider, E. (1999): La TI-92 dans l' enseignement des mathématiques, des enseignant(e)s découvrent la didactique des mathématiques. In: D. Guin (ed.): Actes du congrès "Calculatrices symboliuqes et gémetriques dans l' enseignement des mathématiques ". Montpellier : IREM.

Sträßer, R. (1992): Student's Constructions and Proofs in a Computer Environment. Occasional Paper 134. Bielefeld: IDM, Universität Bielefeld.

\section{Author}

Thomas Gawlick, Oberstufen-Kolleg, Universität Bielefeld, D-33501 Bielefeld, Germany Email: thomas.gawlick@uni-bielefeld.de 